\title{
Separation of Noncutaneous Epithelia in a Fetus Diagnosed In Utero with Junctional Epidermolysis Bullosa
}

\author{
LYNNE T. SMITH, ALEX W. MILLER, DONNA A. KIRZ, SHERMAN ELIAS, \\ STEVEN BRUMBAUGH, AND KAREN A. HOLBROOK
}

\begin{abstract}
Departments of Biological Structure and Medicine (Dermatology). University of Washington, Seattle, Washington 98195 [L.T.S., S.B., K.A.H.]; Departments of Pathology [A.W.M.] and Obstetrics and Gynecology [D.K.], Rush-Presbyterian-St. Luke's Hospital, Chicago, Illinois 60612; and University of Tennessee-Memphis,
\end{abstract} Tennessee 38103 [S.E.]

\begin{abstract}
This study was undertaken to identify the extent of involvement of cutaneous and noncutaneous epithelia during expression in utero of junctional epidermolysis bullosa. Skin and other organs from a 19-wk estimated gestational age fetus affected with junctional epidermolysis bullosa and from age-matched controls were examined by light and transmission electron microscopy. In the affected fetus, skin samples from different body regions including trunk, leg, arm, and finger all showed some separation at the dermal-epidermal junction in the plane of the lamina lucida. Hemidesmosomes were absent or hypoplastic, whereas anchoring fibrils appeared normal in structure and number. Interfollicular epidermis appeared to have separated easily, whereas some follicles remained anchored in the dermis. Areas of epithelium in the trachea and bronchi had separated, but within the lung parenchyma the epithelium of smaller bronchioles and alveoli remained attached to supporting connective tissue. The transitional epithelium of the urinary bladder showed small areas of separation compared with the gall bladder epithelium, which showed extensive separation. Gall bladder epithelium in several control fetuses also was consistently separated. In the affected fetus as well as in controls, gall bladder had multiple layers of basal lamina, a previously unrecognized structural feature of human fetal gall bladder. Epithelia that remained intact included the linings of the stomach and small and large intestines and endothelium of large and small vessels in all organs. Kidney, spleen, liver, and lymph nodes appeared normal in all aspects. In control samples, neither skin nor most of the noncutaneous epithelia had separated from the underlying connective tissue. The epithelial-mesenchymal separations observed in the affected fetus indicate that the abnormality is expressed during fetal development and affects the epithelia of certain internal organs as well as the skin. (Pediatr Res 31: 561 $566,1992)$
\end{abstract}

\section{Abbreviations}

EB, epidermolysis bullosa

JEB, junctional epidermolysis bullosa

DEJ, dermal-epidermal junction

LM, light microscopy

Received July 29, 1991; accepted January 28, 1992

Correspondence: Lynne T. Smith, Department of Biological Structure SM-20, University of Washington, Seattle, WA 98195.

Supported by Dystrophic Epidermolysis Bullosa Research Association of America and National Institutes of Health (HD 17664, AR 21557).
TEM, transmission electron microscopy

EGA, estimated gestational age

EB includes a group of inherited blistering disorders that are categorized according to clinical and morphologic findings into three major subgroups: simplex (or epidermolytic), junctional, and dystrophic (or dermolytic) $(1,2)$. Within each subgroup the severity of the disease and presumably the causal mutation vary. In simplex forms of EB, blisters typically heal without scarring and the plane of blister formation is within or above the basal cell layer of the epidermis. In dystrophic forms of EB, blisters typically heal with scarring; the skin separates at the level of the papillary dermis, deep to the basal lamina of the DEJ. Dystrophic EB is associated with diminution or lack of anchoring fibrils at the DEJ that usually correlates with absent or irregular expression of type VII collagen. In JEB, blisters form within the lamina lucida (3). The epidermal cells remain intact, the lamina densa stays attached to the papillary dermis, and healing of skin lesions occurs without scarring.

JEB is typically inherited as an autosomal recessive disorder. Affected individuals are identified at birth with blisters on various body regions, often with acral involvement (2). Early death may result from fluid loss and sepsis. In postnatal life, patients also exhibit extensive involvement of noncutaneous epithelia, and the consequences of extensive blistering in diverse epithelia adversely affect development and growth. The entire oral cavity, including lips, oral mucosa, tongue, hard and soft palate, and cheeks, may show blistering (4). Tooth development may also be affected by reduced amounts and poor mineralization of enamel and abnormal crown formation. Organs of the digestive system are affected: oropharynx, esophagus, stomach, gall bladder, pancreatic duct, small intestine, large intestine, rectum, and anus (5-7). Involvement of the respiratory system includes the nares, olfactory mucosa, epiglottis, larynx, trachea, bronchi, and bronchioles (8). Alveolar ducts and alveoli appear to be spared. The entire urinary tract can be involved (9). Lesions occur in epithelia of the cervix and vagina and in the external genitalia of both males and females. Structures that appear to be spared include blood vessels, the heart, spleen, liver, and lymph nodes. Involvement of a multiplicity of epithelia compromises the patient's ability to grow normally, and all too frequently an affected individual has a shortened life-span (reviewed in Ref. 10).

Abnormalities in the structure and composition of the hemidesmosomal junctions between the basal keratinocytes and the lamina densa have been implicated as causal for the weakness of the DEJ in JEB (11-13). However, a specific mutation for 


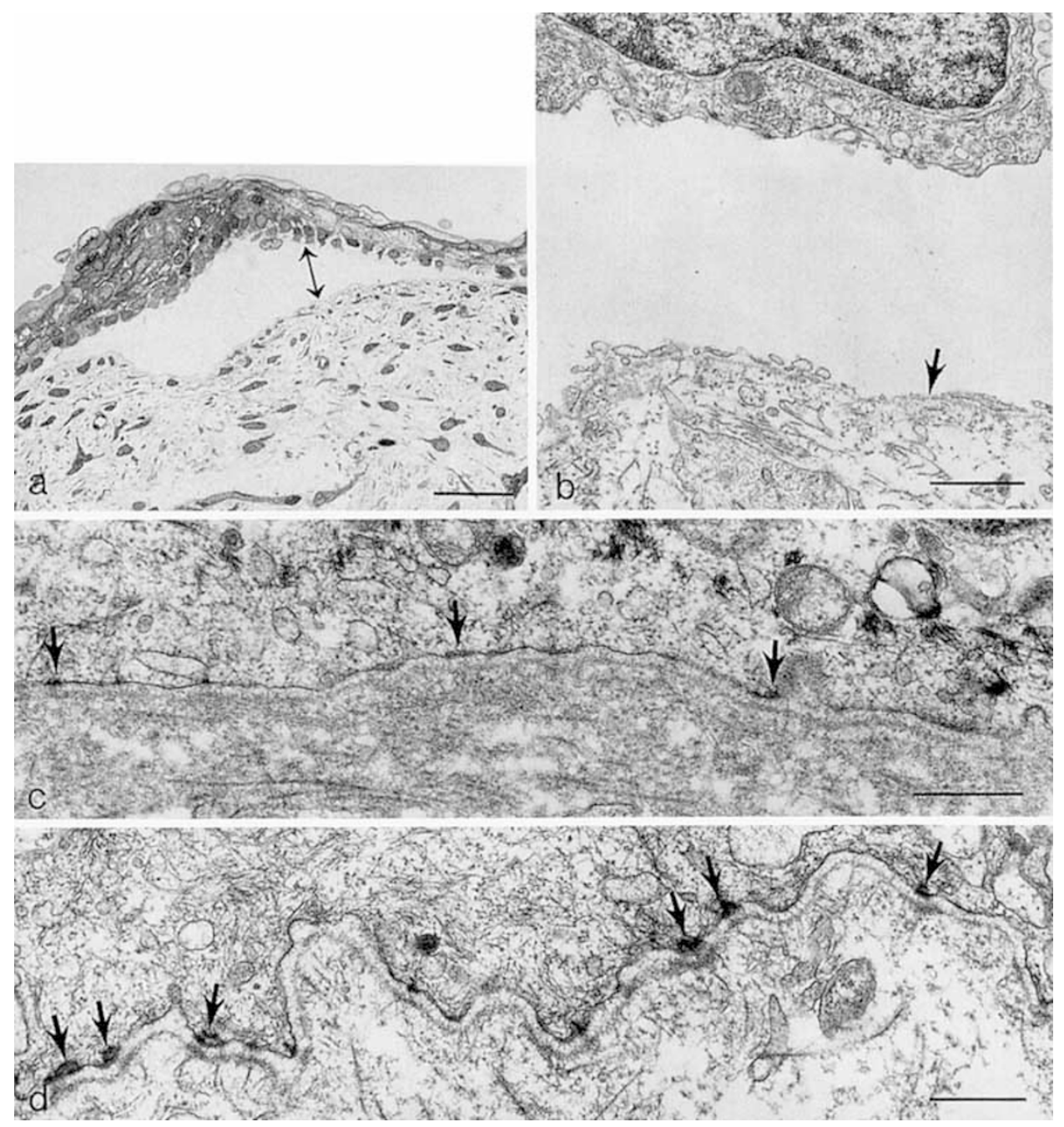

Fig. 1. LM $(a)$ and TEM $(b, c$ and $d)$ of fetal skin biopsies taken by fetoscopy for prenatal diagnosis from fetuses at risk for EB. Skin from the affected fetus showed separation of the epidermis from the dermis, and proteinacous fluid was retained in the cavity ( $a$, double arrow). The lamina densa remained associated with the dermal collagen and formed the floor of the blister cavity $(b$, arrow). Where the epidermis remained attached, some electron-dense material was present, but complete hemidesmosomes were not evident $(c$, arrows). This contrasted with the structure and distribution of hemidesmosomes ( $d$, arrows) in a similar biopsy taken from a normal fetus that was at risk for recessive dystrophic EB. Bar in $a, 50$ $\mu \mathrm{m} ; b, 1 \mu \mathrm{m} ; c$ and $d, 0.5 \mu \mathrm{m}$.

inherited JEB is unknown. Noncutaneous epithelia lack true hemidesmosomes but display hemidesmosome-like structures. Blistering of noncutaneous epithelia suggests that the abnormality in JEB may involve something other than the hemidesmosome per se, possibly an enzyme or factor that relates to or helps to integrate the hemidesmosome structural components.

A 19- to 20-wk-old fetus affected with the Herlitz or "letalis" type of JEB was identified by prenatal diagnosis and at autopsy with cutaneous lesions on many body regions. Although extensive noncutaneous involvement in patients with this type of JEB has been documented, very little has been reported concerning the extent of involvement during fetal life. To identify which tissues might be affected during this early period of development, samples were taken at autopsy from the affected fetus and compared by LM and TEM to similar samples from agematched, non-EB fetuses.

\section{MATERIALS AND METHODS}

A 27 -y-old mother previously delivered a baby affected with JEB who died in the neonatal period. Prenatal diagnosis was performed during the subsequent pregnancy at 18 wk EGA. Two biopsies were obtained by ultrasound-guided fetal skin sampling (14) and evaluated by LM and TEM to reflect an affected fetus (see Results). The parents chose to discontinue the pregnancy.

Samples were obtained from the fetus at the time of autopsy with permission of the family and approval of the Internal Review Board at Presbyterian-St. Luke's Hospital. Comparable tissue samples from two age-matched normal fetuses were obtained through the Central Laboratory for Human Embryology at the University of Washington courtesy of Dr. Thomas Shepard in accordance with USPHS guidelines and with Human Subjects Institutional Review approval.

Tissue samples were placed in half-strength Karnovsky's fixative, postfixed in $1 \%$ osmium tetroxide, en bloc stained in $1 \%$ uranyl acetate, dehydrated through a graded ethanol series and propylene oxide, and embedded in Epon. One- $\mu \mathrm{m}$ thick sections were mounted on glass slides, stained with Richardson's blue (15), and examined and photographed using a Zeiss standard photomicroscope. Ultrathin sections were stained sequentially with $1 \%$ phosphotungstic acid, saturated uranyl acetate, and Reynold's lead citrate (16) and viewed using a Philips 420 transmission electron microscope (Philips Electronic Instruments, Mahwah, NJ) at $60 \mathrm{kV}$. 


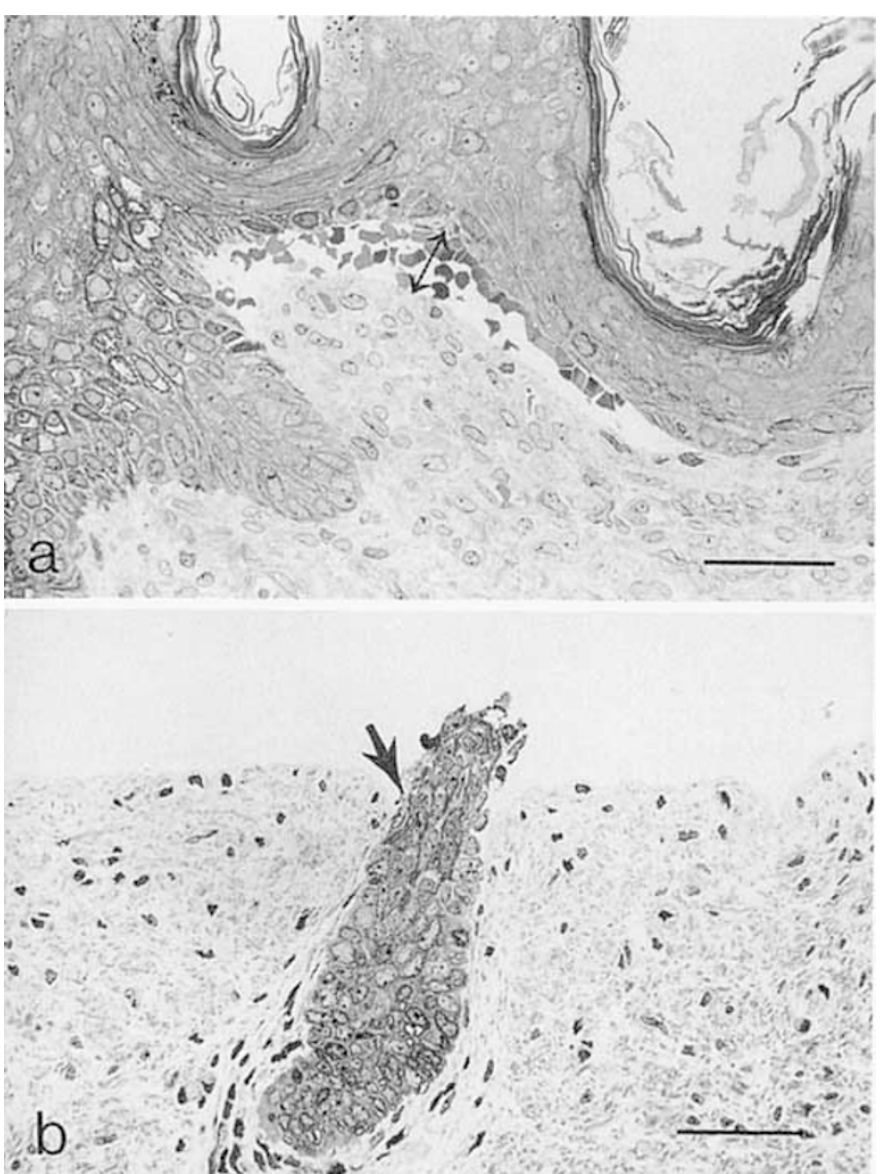

Fig. 2. LM of skin from the fingertip $(a)$ and leg $(b)$. In the fingertip, the epidermis remained associated with the dermis $(a)$, but microblisters (double arrow) were present. Lesional skin on the knee was denuded of epidermis, but hair follicles frequently remained anchored in the dermis (b, arrow). Bar in $a$ and $b=50 \mu \mathrm{m}$.

\section{RESULTS}

Fetal skin biopsy. Of the two fetal skin biopsies obtained through fetoscopy, one sample appeared intact and the other showed clear separation of the epidermis from the underlying dermis (Fig. 1 $a$ ) at the level of the lamina lucida (Fig. 1b). In both samples, hemidesmosomes were sparse, hypoplastic, and lacked the subbasal dense plate (Fig. $1 c$ ). This represented substantially diminished junctional structure compared with that typically seen in fetal skin biopsies from normal fetuses (Fig. 1d).

Gross morphology at autopsy. By gross examination, all organ systems except the skin appeared normal; these included the cardiovascular system, respiratory system, liver and gall bladder, spleen and pancreas, adrenals, genitourinary system, gastrointestinal tract, and skeletal system. The placenta was immature, appropriate for the gestational age of the fetus ( $20 \mathrm{wk}$ ). A fusiform hematoma was present in the midportion of the umbilical cord.

Histologic and ultrastructural findings. Integumentary system. Blisters were present on the occiput, trunk, and upper and lower limbs. By LM, some areas appeared intact, whereas in other areas, such as the fingertip, microblisters were apparent (Fig. 2a). On the knee, most of the epidermis had separated, leaving behind the epidermal appendages anchored in the dermis (Fig. $2 b$ ). The lamina densa remained associated with the dermis, and the basal keratinocyte plasma membrane formed the blister roof. Hemidesmosomes were sparse and, at best, hypoplastic in structure (Fig. $1 b$ ).

Digestive system. Oral cavity. By gross examination, the oral tissue appeared to be unaffected; however, by LM, separation of the stratified squamous, nonkeratinized epithelium was noted at the edges of specimens of lip and tongue. These appeared to be artifactual separations that occurred in tissue handling. However, TEM consistently showed hemidesmosome-like structures in control tissue that were lacking or diminished in lip, tongue, and palate from the affected fetus, indicating fine structural differences between affected and controls.

Gastrointestinal tract. Areas of the esophageal epithelium were separated in the plane of the lamina lucida and showed diminished fine structure of the hemidesmosome. The epithelial linings of the stomach, small intestine, and large intestine all appeared normal by LM (Fig. $3 a$ ). By TEM, the basement membrane zone of the small intestine from control tissue showed hemidesmosome-like structures that were lacking in the affected fetus (Fig. $3 b$ and $c$ ). In some areas of the gall bladder, the tall columnar epithelium had separated (Fig. $4 a$ ). In intact areas, the basement membrane included several layers of basal laminae (Fig. 4c). Where the epithelium had separated, the layers of basal laminae remained associated with the connective tissue of the gall bladder (Fig. 4b). Control fetal gall bladder also showed redundant basal laminae (Fig. 4d).

Respiratory svstem. The pseudostratified, ciliated, columnar epithelium of the trachea was separated in numerous areas, and some blister cavities contained fluid (Fig. $5 a$ ), indicating that lesions may have been present before sampling. In contrast, with the control tissue there was also a lack of hemidesmosome-like structures in the affected fetus (Fig. $5 b$ and $c$ ). Bronchial epithelium also showed areas of separation, but deeper in the respiratory tree (small bronchioles through alveoli) all of the epithelia remained intact.

Urogenital system. The kidney tubules, ducts, and renal calyces appeared normal. The transitional epithelium of the urinary bladder showed areas of separation (Fig. 6a), whereas the epithelia of the ureter and urethra remained intact. Ultrastructurally, the basement membrane zones of ureter, urethra, and urinary bladder in controls showed hemidesmosome-like densities that were reduced or absent in the affected fetus compared with controls (Fig. $6 b$ and $c$ ).

Other systems. The endothelia of the aorta and smaller blood vessels were intact. The spleen, liver, and lymph nodes appeared normal.

\section{DISCUSSION}

In JEB, separation of certain noncutaneous epithelia appears to occur in utero. Although epithelial separations were noted in several organ systems, overall morphology and development of the fetus affected with JEB was apparently normal. The affected sites corresponded to areas of potential abrasion and/or tension at this stage in development (esophagus, trachea, bronchus, and urinary bladder). Other organs or structures that did not show separations (bronchioles, stomach, and intestine) may be more protected from abrasion or mechanical stresses. Normal tissues had hemidesmosome-like structures: there was electron-dense material along the cytoplasmic surface of the basal plasma membrane without a corresponding subbasal dense plate in the underlying lamina lucida. Nonetheless, several of the epithelia that appeared undisturbed in situ displayed reduced hemidesmosome-like structures compared with controls, thus suggesting that with increased fetal activity at later stages of gestation there may be an increased risk of blistering in noncutaneous epithelia.

Hemidesmosomes are defined ultrastructurally as electrondense structures that straddle the basal plasma membranes of keratinocytes along the DEJ and biochemically by the presence of certain antigens. Keratin filaments insert into the attachment plaque; the corresponding subbasal dense plate lies beneath the plasma membrane within the lamina lucida. Anchoring filaments span the lamina lucida zone, concentrated beneath hemidesmosomes. Bullous pemphigoid antigen localizes to the attachment plaque (17), and BM600 is a glycoprotein that localizes to the 

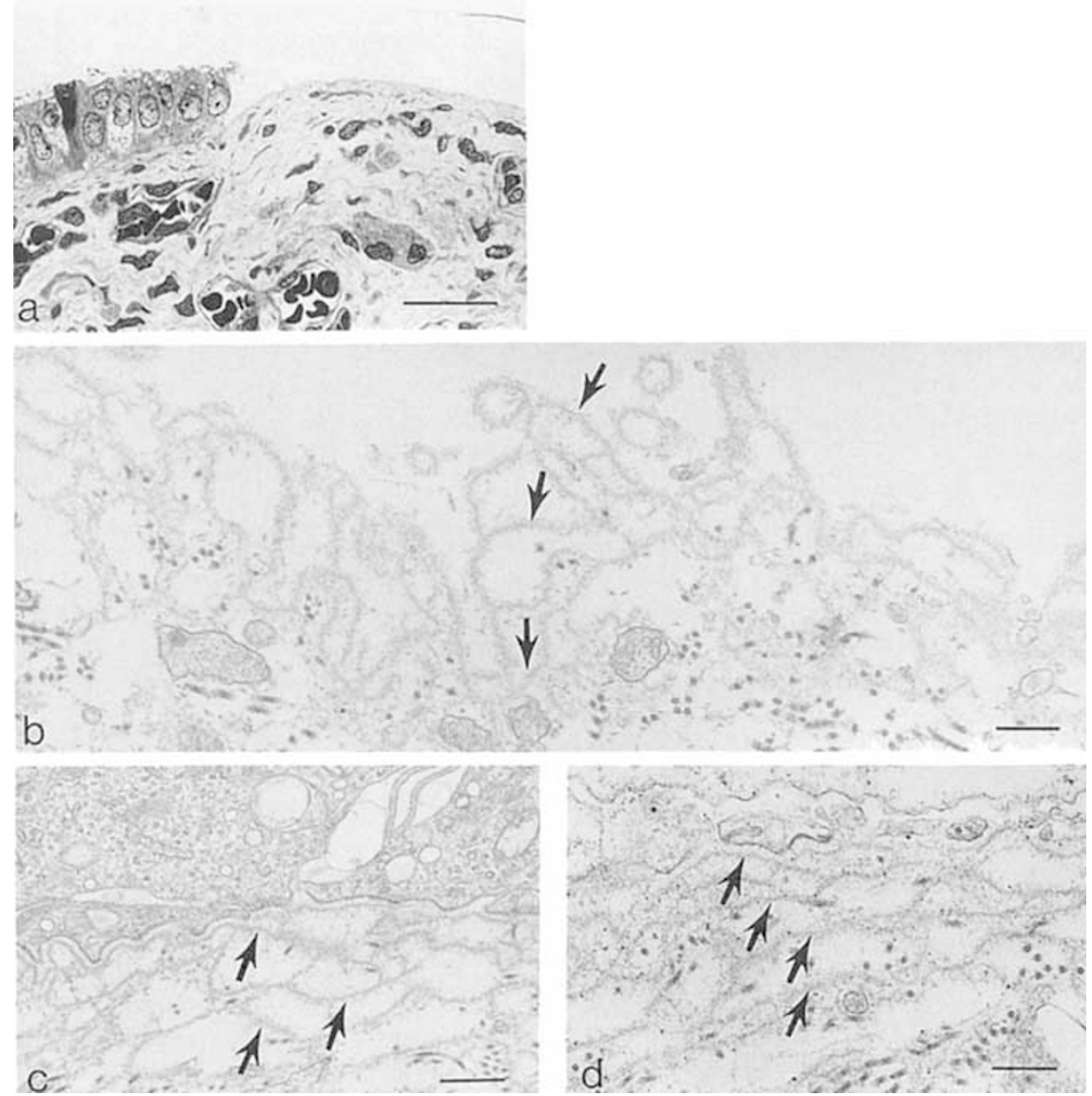

Fig. 3. LM and TEM of the small intestine. By LM, the intestinal epithelium appeared intact $(a)$. Ultrastructurally, the small intestine of the affected fetus ( $b$ ) showed diminished attachment structure compared with the hemidesmosome-like structures (arrows) found in the control $(c)$. Bar in $a, 50 \mu \mathrm{m} ; b$ and $c, 0.5 \mu \mathrm{m}$.

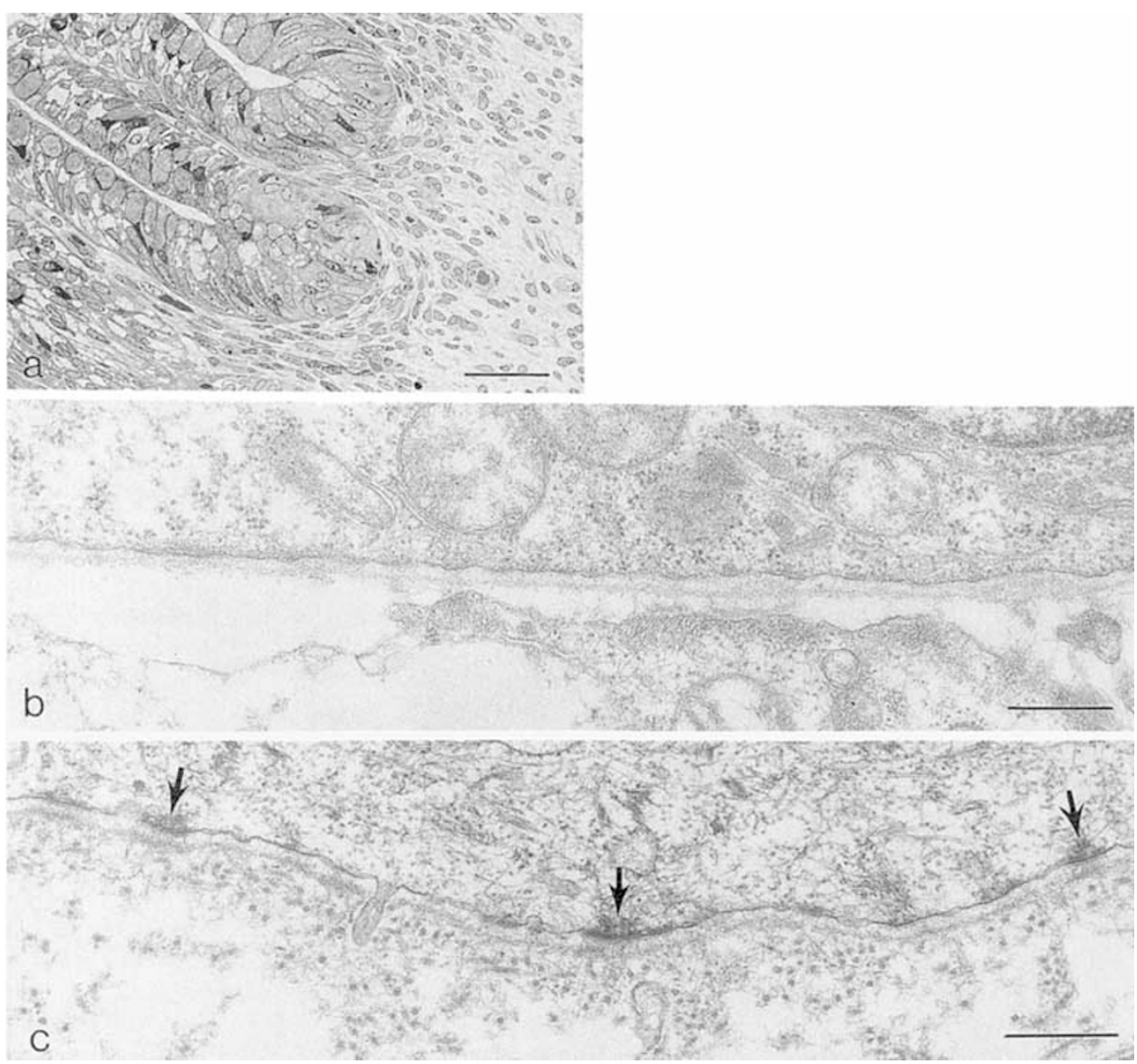

Fig. 4. LM and TEM of gall bladder. The gall bladder $(a)$ showed attached and separated areas. Where the epithelium had lifted $(b)$, several layers of basal laminae (arrows) remained associated with the connective tissue. The intact epithelium of the affected fetus $(c)$ and the control gall bladder showed redundant basal laminae (d, arrows). Bar in $a, 50 \mu \mathrm{m} ; b-d, 0.5 \mu \mathrm{m}$. 


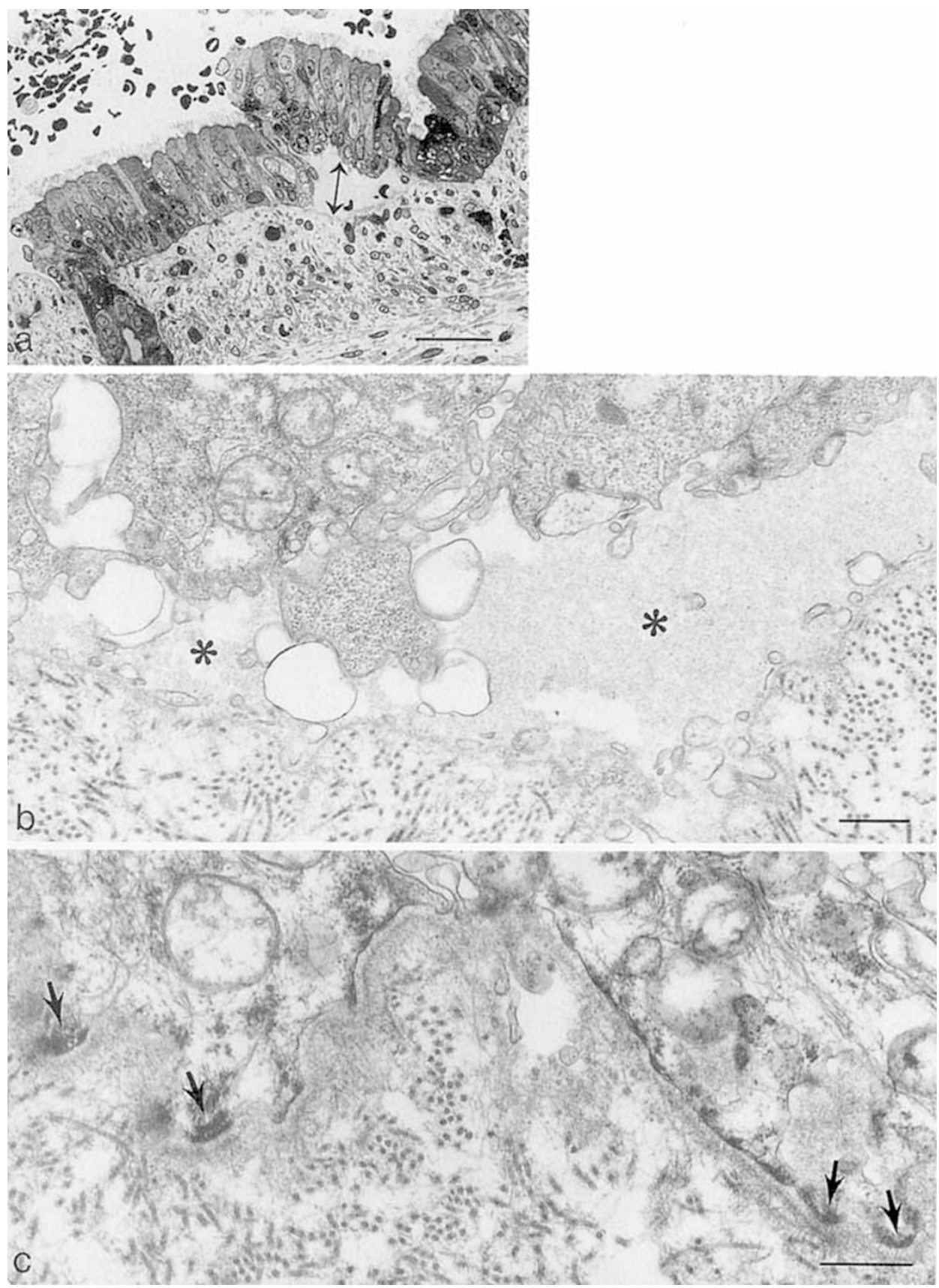

Fig. 5. LM and TEM of trachea. LM showed separation of the epithelium of the trachea (a, double arrow). Ultrastructure of the affected trachea (b) showed fluid in the blister cavity $\left(^{*}\right)$ and diminished junctional fine structure compared with hemidesmosome-like structures (arrows) present in the control trachea (c). Bar in a, $50 \mu \mathrm{m} ; b$ and $c, 0.5 \mu \mathrm{m}$.

lamina lucida and lamina densa zone of the DEJ and is hypothesized to associate with the extracellular component of hemidesmosomes (18). In JEB, part or all of the hemidesmosome may be absent. Material corresponding to the attachment plaque may be present, but the subbasal dense plate typically is absent $(3,13$, 19).

Other studies have compared JEB and controls for the distribution of antigens of the DEJ detected by MAb labeling. GB3 is an MAb that recognizes BM600 (18). Immunolabeling for GB3 in postnatal individuals is absent or diminished in skin from patients with the Herlitz or letalis type of JEB, variably present in other forms of JEB, and consistently expressed in simplex EB and dystrophic EB $(20,21)$. In noncutaneous epithelia, GB3 immunolabeling has been shown to be reduced in JEB compared with normal fetuses in the esophagus. GB3 labeling done in normal fetal tissues, but not in affected fetuses, demonstrated linear, subepithelial staining of GB3 in the stomach, small intestine, colon, urinary bladder, bronchioles, and gall bladder (20). In disagreement with these data, Nazzaro et al. (22), however, report no GB3 labeling in normal stomach of an 18-wk EGA fetus. In these same tissues, we have found differences in the morphology of hemidesmosome-like structures between the normal and the JEB-affected fetus. It remains to be determined whether the compromised structure also reflects changes in antigen expression.

The affected fetus that we studied differed from a recently reported case of a fetus diagnosed as having JEB with pyloric atresia (22). In our case, the pylorus had a patent lumen and normal epithelial adherence to the mesenchyme, indicating absence of pyloric atresia. In contrast, the pyloric lumen of the fetus reported by Nazzaro et al. (22) was filled with connective tissue and apparently lacked the mucosa. Our studies agree in 


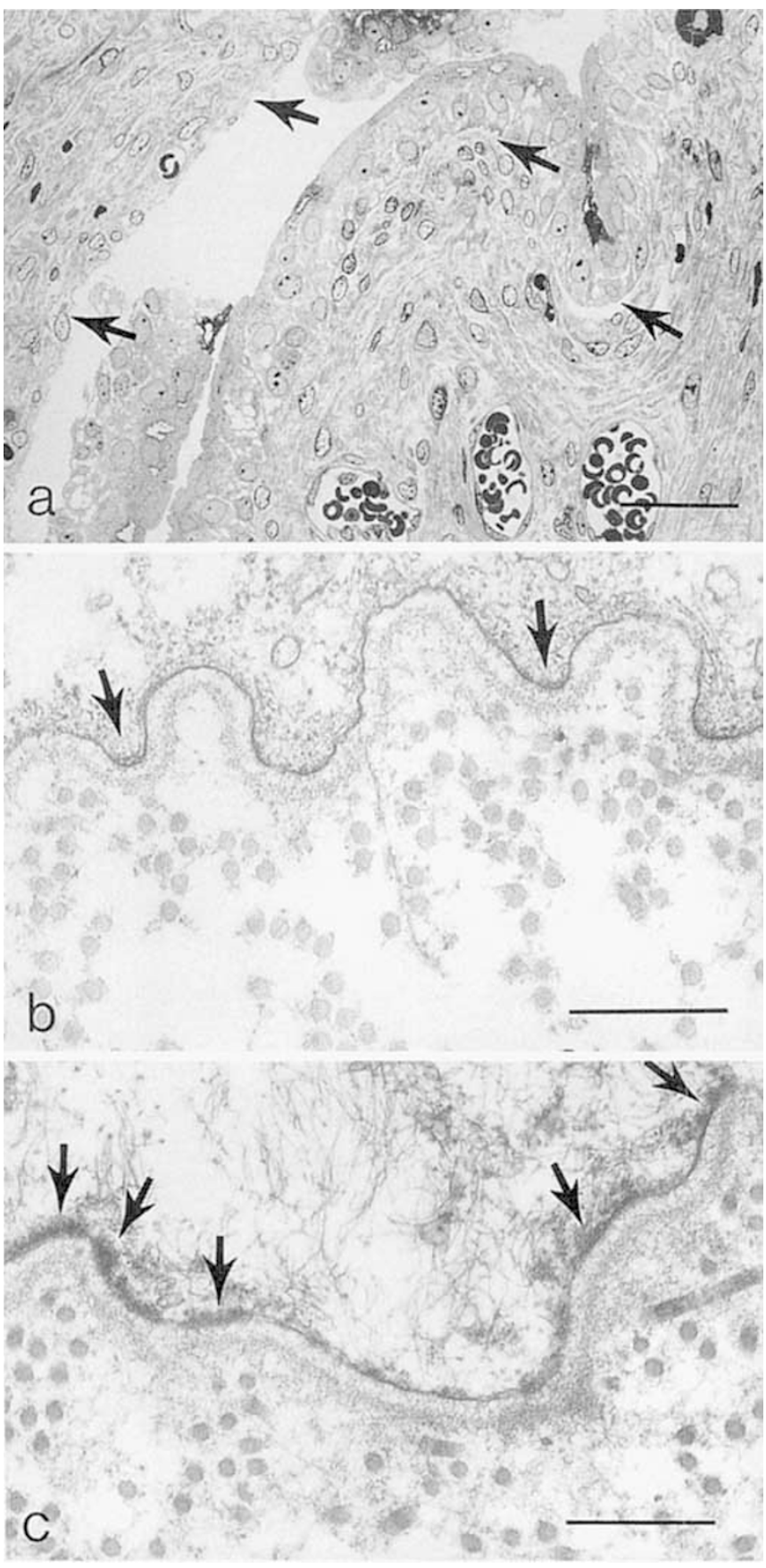

Fig. 6. LM of urinary bladder and TEM of ureter. Separation of the epithelium is apparent in the urinary bladder ( $a$, arrows). TEM of the ureter revealed a reduced amount of structure (arrows) in the basal compartment of the epithelium in the affected $(b)$ compared with the control (c). Bar in $a, 50 \mu \mathrm{m} ; b$ and $c, 0.5 \mu \mathrm{m}$.

finding diminished hemidesmosome-like structures in the stomach of the affected fetuses compared with controls.

A new finding in the present study was the presence of redundant basal laminae underlying the gall bladder epithelium in the affected and the normal fetuses at $20 \mathrm{wk}$. At birth, there is a single basal lamina underlying the gall bladder epithelia in nor$\mathrm{mal}$ infants and in infants affected with JEB (19). Redundant basal laminae are normally present around postcapillary venules in the skin and beneath other epithelia, where they correlate with injury and repair of tissue. Bile acids are very low before $17 \mathrm{wk}$ EGA, when there is a surge of bile synthesis, and thereafter concentrations continue to increase (23). The occurrence of redundant basal laminae in developing gall bladder may reflect a combination of the state of rapid synthesis of basement membrane components, the relative fragility of fetal structures, and a transient abundance of bile acids in the fetal gall bladder.

The ultrastructural findings of this study demonstrate diminished attachment structures at epithelial-mesenchymal boundaries in several noncutaneous epithelial as well as in the skin of a fetus affected with JEB. The otherwise normal appearance of all organs that we observed supports the notion that in the protected environment of the womb the fetus affected with JEB undergoes normal development.

Acknowledgments. The authors thank Mary Hoff and Marcia Usui for expert technical assistance.

\section{REFERENCES}

1. Gedde-Dahl T 1981 Sixteen types of epidermolysis bullosa. Acta Derm Venereol [Suppl] (Stockh) 95:74-87

2. Haber RM, Hanna W Ramsay CA, Boxall TBH 1985 Hereditary epidermolysis bullosa. J Am Acad Dermatol 113:252-278

3. Pearson RW, Potter B, Strauss F 1974 Epidermolysis bullosa hereditaria lethalis. Arch Dermatol 109:349-355

4. Nowak AJ 1988 Oropharyngeal lesions and their management in epidermolysis bullosa. Arch Dermatol 124:742-745

5. Leland LS, Hirschi D 1954 Epidermolysis bullosa hereditaria letalis in newborn twins. Am J Dis Child 87:321-327

6. Gryboski JD, Touloukian R, Campanella RA 1988 Gastrointestinal manifestations of epidermolysis bullosa in children. Arch Dermatol 124:746-752

7. Orlando RC, Bozymski EM, Briggaman RA 1974 Epidermolysis bullosa: gastrointestinal manifestations. Ann Intern Med 81:203-206

8. Maddison TG, Barter RA 1961 Epidermolysis bullosa hereditaria letalis. Arch Dis Child 36:337-339

9. Kretowski RC 1973 Urinary tract involvement in epidermolysis bullosa. Pediatrics 51:938-941

10. Holbrook KA 1988 Extracutaneous epithelial involvement in inherited epidermolysis bullosa. Arch Dermatol 124:726-731

11. Fine JD, Holbrook KA, Elias S, Anton-Lamprecht I, Rauskolb R 1990 Applicability of 19-DEJ-1 monoclonal antibody for the prenatal diagnosis on exclusion of junctional epidermolysis bullosa. Prenat Diagn 10:219-229

12. Hashimoto I, Anton-Lamprecht I, Neuberg P 1975 Epidermolysis bullosa hereditaria letalis: report of a case and probable ultrastructure defects. Hel Paediatr Acta 30:543 552

13. Tidman MJ, Eady RAJ 1986 Hemidesmosome heterogeneity in junctiona epidermolysis bullosa revealed by morphometric analysis. J Invest Dermato $86: 51-56$

14. Shulman LP, Elias S 1990 Percutaneous umbilical cord blood sampling, feta skin sampling and fetal liver biopsy. Semin Perinatol 14:456-464

15. Richardson KC Jartett L, Finke EH 1960 Embedding in epoxy resins fo ultrathin sectioning in electron microscopy. Stain Technol 35:313-323

16. Reynolds ES 1963 The use of lead citrate at high $\mathrm{pH}$ and an electron opaqu stain in electron microscopy. J Cell Biol 7:208-213

17. Furue M, Iwata M, Tamaki K, Ishbashi Y 1986 Anatomical distribution an immunological characteristics of epidermolysis bullosa acquisita antigen anc bullous pemphigoid antigen. Br J Dermatol 114:651-659

18. Verrando P, Blanchet-Bardon C. Pisani A, Thomas L, Cambazard F, Ead RAJ, Schofield O, Ortonne JP 1991 Monoclonal antibody GB3 defines : widespread defect of several basement membranes and a keratinocyte dys function in patients with lethal junctional epidermolysis bullosa. Lab Inves 64:85-92

19. Schnachner L, Lazarus GS, Dembitzer H 1977 Epidermolysis bullosa heredi taria letalis. Pathology, natural history and therapy. Br J Dermatol 96:51-58

20. Verrando P. Hsi BL Yeh CI, Pisani A Serieys N Ortonne JP 1987 Monoclona antibody GB3, a new probe for the study of human basement membrane: and hemidesmosomes. Exp Cell Res 170:116-128

21. Schofield OMV, Fine JD, Verrando P, Haegerty AHM, Ortonne JP, Eady RA. 1990 GB3 monoclonal antibody for the diagnosis of junctional epidermolysi bullosa: results of a multicenter study. J Am Acad Dermatol 23:1078-1083

22. Nazzaro V, Nicolini U, De Luca L, Berti E Caputo R 1990 Prenatal diagnosi of junctional epidermolysis bullosa associated with pyloric atresia. J Mec Genet 27:244-248

23. Colombo C. Zuliani G, Ronchi M, Breidenstein J, Setchell KDR 1987 Biliar bile acid composition of the human fetus in early gestation. Pediatr Re 21:197-200 